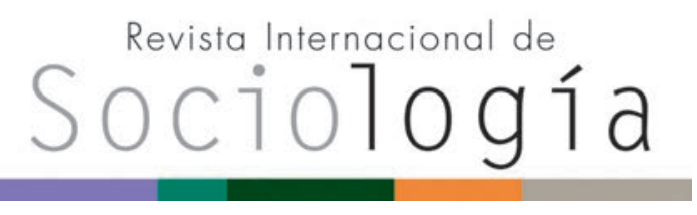

Revista Internacional de Sociología RIS

vol. 77 (2), e123, abril-junio, 2019, ISSN-L:0034-9712 https://doi.org/10.3989/ris.2019.77.2.18.049

\title{
MODELOS DE DINÁMICAS DE LA OPINIÓN. UNA REVISIÓN DE LA LITERATURA
}

\author{
JORDI TENA-SÁNCHEZ \\ Universitat Autònoma de Barcelona \\ jordi.tena@uab.cat \\ ORCID iD: https://orcid.org/0000-0001-8569-5742 \\ FRANCISCO JOSÉ LEÓN-MEDINA \\ Universitat de Girona \\ francisco.leon@udg.edu \\ ORCID iD: https://orcid.org/0000-0003-1713-9757
}

Cómo citar este artículo / Citation: Tena-Sánchez, J. y F. J. León-Medina. 2019. "Modelos de dinámicas de la opinión. Una revisión de la literatura". Revista Internacional de Sociología 77(2):e123. https://doi. org/10.3989/ris.2019.77.2.18.049

\section{RESUMEN}

El estudio de la opinión pública está girando, en los últimos años, del tradicional enfoque descriptivo con fınes in-formativos y de asesoramiento a un nuevo enfoque expli-cativo y generativista. Este nuevo enfoque se ha centrado en la construcción y análisis de modelos en los que las interacciones locales, microscópicas, generan las regula-ridades macroscópicas de la opinión pública. La fertilidad de este nuevo enfoque se está traduciendo en un ritmo de publicaciones que puede llegar a ser abrumador. En este trabajo presentamos una revisión actualizada de la literatura sobre los modelos de dinámicas de la opinión. Se presentan los principales modelos y sus extensiones, organizado la exposición alrededor de diez ejes de de-bate que configuran el contenido de cada aportación. El artículo ofrece también algunas reflexiones sobre los prin-cipales desafíos que tienen ante sí los científıcos sociales interesados en el estudio de las dinámicas de la opinión.

\section{Palabras Clave}

Explicación; Influencia social; Modelos basados en agentes; Simulación social; Sociofísica.

Copyright: @ 2019 CSIC. Este es un artículo de acceso abierto distribuido bajo los términos de la licencia de uso y distribución Creative Commons Reconocimiento 4.0 Internacional (CC BY 4.0).

Recibido: 23/03/2018. Aceptado: 10/12/2018.

Publicado online: 04/06/2019

\section{ABstract}

The study of public opinion is turning in the last years, the study from the traditional descriptive approach with informative and advising purposes to a new explana-tory and generativist approach. This new perspective has focused on the construction and analysis of mod-els where local, microscopic, interactions generate macroscopic regularities in public opinion. The fertility of this new approach is causing a publication rhythm that can be overwhelming. In this paper, we present an up to date revision of the literature on opinion dynam-ics models. We present the main models and their extensions, and organize the exposition around ten axes that configure the content of each contribution. The paper also offers some reflections on the main chal-lenges for those social scientists that are interested in the dynamics of public opinion.

\section{KEYWORDS}

Agent-based models; Explanation; Social Influence; Social simulation; Sociophysics. 
The statistical physics approach to social dynamics is currently attracting much interest, as indicated by the large and rapidly increasing number of papers devoted to it. The newcomer can easily feel overwhelmed and get lost in the steadily growing flow of new publications. Even for scholars working in this area, it is difficult to keep up on the new results that appear at an impressive pace.

(Castellano, Fortunato y Loreto 2009: 593).

\section{INTRODUCCIÓN}

La opinión pública y las dinámicas que la conforman constituyen un tema de la mayor relevancia para las ciencias sociales, tanto por su interés a nivel estrictamente académico, como por su trascendencia social y política. En este sentido, el estudio de las dinámicas de opinión es uno de los principales campos de extensión de los modelos formales en las ciencias sociales fuera de la economía $y$, más concretamente, de los modelos de simulación social. La cantidad de modelos de simulación desarrollados en los últimos años pueden hacer que, como sostienen Castellano, Fortunato y Loreto en la cita que sirve de entradilla a este trabajo ${ }^{1}$, el lector, incluso aquel familiarizado con el campo, tenga serias dificultades para mantenerse al día de las nuevas publicaciones. Se trata, por tanto, de un ámbito en el que se han ido publicando periódicamente algunos excelentes trabajos de revisión de la literatura, ya sea sobre modelos de dinámicas de opinión en general o sobre algún tipo de modelo en particular (Abelson 1964; Castellano, Fortunato y Loreto 2009; Flache et al. 2017; Galam 2008; Lorenz 2007; Sîrbu et al. 2007). El objetivo de este artículo es ofrecer una revisión de la literatura que presenta algunas características novedosas. En primer lugar, la mayoría de trabajos de revisión tienen ya una década, lo que hace necesaria una nueva actualización. En segundo lugar, la nuestra es, hasta donde nosotros sabemos, la única revisión existente en español, de manera que hace más fácilmente accesible esta literatura al lector hispanohablante. En tercer lugar, a diferencia del resto de trabajos, el nuestro deja de lado, en la medida de lo posible, los aspectos más técnicos de los modelos y se centra en las cuestiones teóricas más sustantivas, haciendo, una vez más, accesible esta literatura a un público más amplio que la pequeña comunidad estrechamente familiarizada con la modelización formal. En cuarto lugar, nuestra revisión (a excepción de un apartado inicial) tampoco sigue la estructura habitual y los modelos no se agrupan en familias, sino que se ordenan en torno a temas y ejes de debate. Creemos que esta aproximación resulta más fértil de cara a situar los diferentes modelos que la habitual clasificación en familias, pues el flujo actual de publicaciones ha ido dejando más y más desdibujada aquella clasificación. Finalmente, cabe insistir en que, pese a que se hará referencia a los modelos matemáticos, nuestro trabajo se centra en los modelos de simulación social, pues, en general, puede argumentarse que los iniciales modelos matemáticos han dado paso al dominio casi absoluto de los modelos basados en agentes.

El resto del trabajo se organiza como sigue. En el siguiente apartado se realiza un brevísimo repaso histórico de las principales familias de modelos, pues una breve aproximación histórica tiene un valor propedéutico que permite situar mejor las discusiones posteriores. A continuación, se presentarán diez ejes del debate actual alrededor de los cuales se irán presentando los principales rasgos de los modelos de dinámica de la opinión. Los ejes de análisis están organizados alrededor de cinco características clave de todo modelo: los agentes (características de sus opiniones, memoria y otras propiedades individuales relevantes), el tipo de interacción (el rol de la homofilia, el mecanismo de cambio de opinión como resultado de la interacción, la falsificación de preferencias en la interacción y la opinión de referencia que influye en cada agente), el entorno en el que se produce la interacción (el rol de los medios de comunicación), la estructura relacional de partida (las topologías de los modelos) y los resultados que cada modelo produce. Para finalizar, abordamos los principales avances que se han producido y los desafíos que la ciencia social tiene planteados en este terreno.

\section{Principales familias de modelos}

El punto de partida de la discusión académica moderna en torno a los procesos de conformación de la opinión pública podría situarse en la teoría de la aguja hipodérmica de Lasswell (1927). Desde este punto de vista, se consideraba que la opinión pública se formaba a través de la influencia ejercida sobre los ciudadanos por los medios de comunicación y los líderes de opinión. Los ciudadanos eran concebidos como sujetos pasivos que interiorizaban el discurso recibido. Dicha aproximación negligía, además, el papel de la interacción y de la influencia social.

Con los años se fue poniendo de manifiesto lo limitado de esta concepción, señalando, por un lado, que los individuos tienen capacidad de agencia y de resistencia a la influencia ejercida por los medios $y$, por el otro, de manera más importante, que la opinión pública se configura en parte también como resultado emergente de un proceso dinámico de influencia social en interacciones locales. En este sentido, como es bien sabido, el estudio de la emergencia plantea una dificultad para las ciencias sociales, ya que estos fenómenos no pueden deducirse simplemente de las propiedades de los individuos que los generan, de manera que, para poder explicarlos, es imprescindible contar con algún tipo de modelo generativo (Hedström, 2005; 2006). ${ }^{2}$ 
Los primeros modelos formales en este campo fueron modelos matemáticos que se preocuparon, básicamente, por la emergencia del consenso (Abelson 1964; Berger 1981; De Groot 1974; French 1956). No obstante, pese a que hoy en día continúan apareciendo importantes modelos matemáticos (Galam y Javarone 2016$)^{3}$, cada vez existe más acuerdo en torno a las limitaciones de este tipo de modelos, especialmente a su necesaria fundamentación en supuestos falsos (Elster 2007, Hedström 2005; 2006).

En cambio, en los últimos años ha empezado a popularizarse el uso de los modelos de simulación, siendo el estudio de las dinámicas de opinión pública uno de los campos más fructíferos en este sentido. ${ }^{4}$ Los modelos de simulación, y en particular los modelos multi-agente (ABM, por sus siglas en inglés), son una herramienta flexible, idónea para estudiar fenómenos emergentes como la conformación de la opinión pública. Estos modelos permiten introducir agentes con características heterogéneas y realistas (sin tener que recurrir, por ejemplo, al agente representativo típico de los modelos matemáticos basados en la teoría de la elección racional), así como situar a dichos agentes en una estructura o topología de interacción, definida también de forma realista, de manera que los resultados macroscópicos del modelo emergen de la interacción a nivel local, a diferencia, por ejemplo, de los modelos matemáticos basados en la teoría de la elección racional.

Por otro lado, las dinámicas de opinión han constituido en las últimas décadas uno de los grandes campos de expansión de la sociofísica, una rama de la física de sistemas complejos centrada en el análisis de la emergencia de fenómenos sociales. Del seno de esta tradición provienen las familias de modelos más conocidos ${ }^{5}$. Los primeros que se aplicaron a este campo fueron los modelos binarios ${ }^{6}$, como el voter model (Clifford y Sudbury 1973; Holley y Liggett 1975), el Sznajd model (Stauffer 2003; Sznajd-Weron 2005; SznajdWeron y Sznajd 2000) o el majority rule model (Galam 2002) entre otros, aunque rápidamente aparecieron los primeros modelos continuos, los bounded confidence models, entre los que destacan el modelo Deffuant o Deffuant-Weisbuch (Deffuant et al. 2000; Weisbuch et al. 2002; 2003) y el Hegselmann-Krause (Krause 2000; Hegselmann y Krause 2002). A continuación, se presentarán brevemente las características principales de dichos modelos ${ }^{7}$.

Voter model. En este modelo, los agentes están colocados en una cuadrícula. Cada casilla representa a un agente que puede estar en dos estados alternativos (puede tener dos opiniones) ( $(11)$. Los agentes tan solo pueden interactuar con sus vecinos adyacentes. La dinámica del modelo es también extremadamente simple. En cada ronda se forman parejas de agentes. Si ambos agentes comparten el mismo estado, nada sucede; de lo contrario, uno adopta el estado del otro.
Majority rule model. Como en el caso anterior, los agentes en este modelo pueden tener dos opiniones $( \pm 1)$. En este caso, no obstante, cada agente puede comunicarse con cualquier otro agente de la población. En cada ronda se forman grupos de agentes (el tamaño de los grupos varía de una ronda a otra). En cada ronda, todos los agentes adoptan la opinión mayoritaria dentro de su grupo.

Sznajd model. En su versión más simple, los agentes están colocados en una matriz de una única dimensión (una cadena lineal). Una vez más, cada agente puede tener una de dos opiniones $( \pm 1)$. En cada ronda se forman parejas de agentes adyacentes. Si ambos agentes tienen la misma opinión, sus dos vecinos adyacentes a izquierda y derecha se contagian de la misma. En cambio, si discrepan, cada agente le contagia su opinión al vecino del otro.

Deffuant model. Los modelos sociofísicos continuos introducen la cuestión de la homofilia. Se asume la plausible idea de que las personas no suelen interactuar (o, si interactúan, no suelen dejarse influir) por aquellas personas que albergan opiniones muy diferentes de la suya. En el Deffuant, los agentes están enraizados en una determinada estructura de red. Cada agente solo puede interactuar con los agentes a los que está conectado. En cada ronda se forman parejas de agentes. Ahora bien, en estos modelos, además de la opinión (un valor asignado al azar del intervalo —continuo- $[0,1])$, los agentes están dotados de un confidence level que hace las veces de umbral. Si la diferencia de opinión entre ambos agentes excede su confidence level, no sucede nada. De lo contrario, sus opiniones se aproximan en alguna medida (habitualmente, ambos agentes convergen en la media de sus opiniones).

Hegselmann-Krause model. Finalmente, el Hegselmann-Krause es un modelo que trata de capturar la misma idea que el Deffuant a partir de una regla de actualización de creencias muy diferente. En este caso, en cada ronda los agentes interactúan con todos los agentes de la población que queden dentro de su confidence level. Como resultado del encuentro, todos los agentes en un grupo pasarán a compartir la misma opinión, que será la media de las opiniones previas de todos los agentes en el grupo.

Los que acaban de presentarse son, como se ha dicho, los trabajos seminales de las más importantes familias de modelos sociofísicos. En los últimos años, la investigación se ha extendido en múltiples direcciones. Por un lado, existen multitud de desarrollos de esos modelos (por ejemplo, en diferentes tipos de redes). Por otro lado, dentro de la comunidad sociofísica han aparecido nuevos modelos, de última generación, que no son inmediatamente ubicables en una u otra familia (para una presentación detallada hasta el 2009, ver Castellano, Fortunato y Loreto 2009). Y, en tercer lugar, de manera si cabe más importante, 
los modelos de dinámicas de opiniones no se circunscriben en exclusiva a la tradición sociofísica. Algunos de ellos podrían situarse más bien en la senda de la moderna ciencia social analítica y generativa (Axelrod 1997; Baldassarri y Bearman 2007; Duggins 2017). Este tipo de sociología (Hedström 2005, 2006; Hedström y Bearman 2009; Linares 2018) comparte con la sociofísica el proyecto de explicar los fenómenos sociales macroscópicos como efecto de interacciones locales entre entidades microscópicas (agentes o actores, preferentemente individuos), pero se diferencia de la sociofísica en su voluntad de modelizar entidades dotadas de mecanismos cognitivos y de acción plausibles, realistas. Como se ha dicho más arriba, en lo que sigue se discutirán diferentes temas y ejes de debate actuales en el ámbito de los modelos de dinámicas de opinión, presentando las principales contribuciones en cada caso.

\section{CARACTERÍSTICAS DE LAS OPINIONES}

\section{Modelos con variables de opinión binarias; discretas con más de dos opciones; conti- nuas, y mixtos}

En muchos modelos la opinión de los agentes se modeliza a partir de una variable binaria (típicamente, \pm 1 ). Son de este tipo los modelos binarios clásicos citados más arriba, así como muchos modelos de última generación (Bartolozzi, Leinweber y Thomas 2005; Dall'Asta y Castellano 2007; Gil y Zanette 2006; Grabowsky y Kosinsky 2006; Halu et al. 2013; Jarman et al. 2015; Jiang, Hua y Chen 2007; Jiang et al., 2008; Nowak, Szamrej y Latané 1990; Sabatelli y Richmond 2003, 2004; Sobkowicz 2003, 2010; Sousa 2005). Los modelos binarios no están, ni mucho menos, en desaparición. Los bounded confindence models clásicos y la mayoría de modelos actuales son, empero, continuos (Baldassarri y Bearman 2007; Dandekar, Goel y Lee 2013; Duggins 2017; Fortunato 2004, 2005; Gargiulo y Gandica 2017; Kurahashi-Nakamura, Mäs y Lorenz 2016; Lallouache, Chakraborti y Chakrabarti 2010a, 2010b; Marsden 1981; Mäs, Flache y Helbing 2010; Mavrodiev, Tessone y Schweitzer 2013; Moussaïd et al. 2013; Sen 2011, 2012; Shin y Lorenz 2010; Smaldino y Epstein 2015; Sousa 2004).

Desde nuestro punto de vista, no existen razones para preferir uno u otro tipo de modelo, en general, y la elección debería depender del tema concreto que quiera estudiarse. Así, no cabe duda de que, en multitud de casos, una variable continua puede ser la forma más adecuada de representar las opiniones de las personas (por ejemplo, la ideología política suele conceptualizarse como un continuum que va desde la extrema izquierda a la extrema derecha), mientras que una variable binaria puede ser la opción más adecuada en muchos otros. Numerosas e importantes conductas y temas de discusión son del tipo "blanco o negro": fumar o no, vestir un burka o no hacerlo, manifestar nuestra discrepancia con el régimen en una dictadura o no hacerlo, etc.

Existen también modelos discretos con más de dos opciones (normalmente con tres) (Balenzuela, Pinasco y Semeshenko 2015). Muchos son derivaciones de los sociofísicos binarios clásicos. Por ejemplo, Mobilia (2011) presenta un voter model con tres opciones y Baker y Hague (2008), Schulze (2004) o Sousa (2004) presentan variaciones discretas del Sznajd (ver también Behera y Schweitzer 2003; Bernardes et al. 2001, 2002). Algunos también introducen la confianza limitada (bounded confidence). Por ejemplo, hay versiones bounded confidence discretas con tres opciones del voter model (Vázquez, Krapivsky y Redner 2003), del majority rule model (Gekle, Peliti y Galam 2005) o del Sznajd model (Stauffer 2002). También hay modelos que representan el camino contrario, variaciones discretas de modelos continuos clásicos. Por ejemplo, Stauffer, Sousa y Schulz (2004) desarrollan una versión discreta del Deffuant y Fortunato (2004) o Vázquez, Krapivsky y Redner (2003) desarrollan versiones discretas del Hegselmann-Krause.

Finalmente, existe un interesante, aunque minoritario, tipo de modelos que quizá podríamos calificar de mixtos, en el que los agentes albergan al mismo tiempo una variable de opinión continua y otra binaria. Los más célebres son los modelos sociofísicos del tipo CODA (Continous Opinions and Discrete Actions). En el modelo seminal de Martins (2008), por un lado, los agentes tienen una opinión que es continua, privada y no observable; mientras que, por el otro, desarrollan una conducta que es binaria, pública y observable. Como argumenta Martins, ciertamente muchas importantes actitudes toman una forma binaria de "sí o no" (manifestarse contra el régimen, por ejemplo), pero eso no es óbice para reconocer que cada persona puede estar internamente más o menos convencida de su apoyo al sí o al no. Así, los agentes del modelo pueden ir cambiando de opinión de forma gradual hasta que, sobrepasado un determinado umbral, cambian de conducta.

\section{Modelos mono o multitema}

La gran mayoría de modelos existentes son monotemáticos, a saber, solo existe un tema sobre el que los agentes tienen opinión. No obstante, también existen algunos modelos multitemáticos, en los que los agentes albergan opiniones sobre una variedad de temas (Fortunato et al. 2005; así como una compilación de referencias en Lorenz 2007:14 y Sîrbu et al. 2007: 2.2). Como sostiene Sobkowicz (2009: 2.10) esta innovación permite dotar de mayor riqueza y realismo a los modelos. Particularmente interesante es el modelo de Baldassarri y Bearman (2007). Dicho modelo caracteriza de manera más 
realista el supuesto clásico de los modelos bounded confidence: cuando dos agentes con una opinión muy diferente sobre un determinado tema se encuentran, no se abstienen de interactuar, sino que disponen de la opción de no hablar sobre ese tema, pero sí de algún otro.

\section{ELPROBLEMADELAMEMORIAENLOSMODE- LOS BINARIOS}

En los modelos sociofísicos binarios clásicos los agentes no tienen memoria de las interacciones pasadas (Castellano, Fortunato y Loreto 2009, Sîrbu et al 2007, Lorenz 2007). La ausencia de memoria implica que la opinión de un agente en $t_{n}$ no afecta en absoluto a su opinión en $t_{n+1}$. Un agente que opina +1 en $t_{n}$ puede pasar a opinar - 1 en $t_{n+1}$ con base en una simple interacción con otro agente. Y, aún más, con el paso del tiempo su opinión puede ir cambiando de un extremo al otro sin ningún límite.

Esto es, obviamente, poco irrealista. Existe abundantísima evidencia empírica de la existencia de lo que Kuran (1987b; 1988; 1995: 171) denominaba "perseverancia de las creencias": una vez que una persona se ha formado una creencia es relativamente difícil que esta varíe, aunque dicha persona vea que la opinión pública está cambiando, aparezca nueva evidencia contraria a dicha creencia, etc.

En los últimos tiempos, empero, han surgido algunos modelos que tratan de solventar este problema. La dinámica de cambio de la conducta pública a partir de transformaciones graduales en la opinión privada (en los modelos CODA) debe entenderse como una interesante propuesta en este sentido. Así, por ejemplo, Dall'Asta y Castellano (2007) elaboran un desarrollo del voter model en el que dotan de memoria a los agentes a través de un procedimiento similar al de Martins (2008). Los agentes tienen un umbral para cambiar de opinión (¿cuántas interacciones tengo que tener con agentes de opinión contraria para cambiar yo de opinión?). Así, un agente solo cambia de opinión después de un cierto número de interacciones con agentes de opinión contraria.

\section{DISTINCIÓN ENTRE OPINIÓN E INFLUENCIA}

En los primeros modelos sociofísicos, los agentes eran definidos por una sola característica: su opinión ${ }^{8}$. En cambio, hoy en día prácticamente todos los modelos incluyen agentes definidos también por alguna o algunas otras variables relacionadas con la influencia social. Como sostiene Sobkowicz (2009: 2.10), esta innovación es una mejora general del realismo de los modelos que abre la puerta a asignar roles y poderes diferenciados a los agentes. A continuación, se discuten brevemente algunas de esas características típicas.

\section{Persuasiveness y suportiveness}

Muchos modelos incorporan diferentes grados de persuasiveness (capacidad de hacer cambiar a otros de opinión) y de suportiveness (capacidad de reforzar la opinión de otros que ya piensan como uno mismo). Los primeros modelos que incorporaban estos parámetros se basaban en la teoría del impacto social de Latané (1981), aunque hoy en día muchos modelos incorporan estas características (por ejemplo, Grabowsky y Kosinsky 2006; Laguna et al. 2005; Nowak, Szamrej y Latané 1990; Nowak y Lewenstein 1996; Sen 2011; y, para una revisión de estos modelos, ver Holyst, Kacperski y Schweitzer 2001).

\section{Estatus}

Curiosamente, no existen demasiados modelos que tomen en consideración el papel que pueden jugar las jerarquías de estatus en las dinámicas de opinión pública. Laguna et al. (2005) y Grabowsky y Kosinsky (2006) son dos de las escasas excepciones en este sentido. En estos modelos, los agentes de más estatus son más influyentes y menos influenciables que los de estatus bajo.

Pese a que la escasez de modelos que toman en consideración explícitamente el papel de las jerarquías de estatus puede resultar sorprendente, también es cierto que muchos otros modelos que incluyen otras características, como la persuasiveness, están, de hecho, capturando la misma idea con un término distinto. En este sentido, por ejemplo, Crokidakis y Oliveira (2011) presentan una variación del Sznajd model en el que los agentes se ordenan jerárquicamente en función no de su estatus, sino de su reputación. Sin embargo, la idea es claramente la misma. Una díada de agentes puede influir a sus vecinos solo si la reputación media de la díada es mayor que la de los vecinos. Y aún en una línea similar, Barth (2006) presenta un modelo en el que unos agentes (que, en este caso, son empresas) tienen más autoridad que otros.

\section{Compromiso}

En multitud de modelos los agentes tienen diferentes grados de compromiso con su opinión, a saber, están más o menos convencidos de la bondad de la misma (Baldassari y Bearman 2007; Balenzuela, Pinasco y Semeshenko 2015; Chacoma y Zanette 2015; Duggins 2017; Lallouache, Chakraborti y Chakrabarti 2010a, 2010b; Martins 2008; Salzarulo 2006; Sen 2011). A partir de aquí, los diferentes modelos asumen tesis progresivamente más fuertes respecto a la importancia del compromiso. Una idea compartida por todos es que a los agentes más comprometidos con sus creencias les cuesta más cambiarlas (son más resistentes a la influencia social). Esta idea ya estaba en los trabajos clásicos de Kuran (1993; 1995: 167 y ss.) quien sostenía (aunque no 
llegó a modelizarlo) que las creencias pueden ubicarse en un continuo desde las creencias soft (construidas con base en la heurística de la prueba social) hasta las hard (construidas a partir de la experiencia, conocimiento experto, etc.). Según Kuran, las creencias hard son más resistentes a la influencia.

La anterior parece una tesis obvia. No obstante, muchos modelos van un paso más allá. Habitualmente, se supone también que los agentes que tienen mayor compromiso con sus creencias tienen más resistencia a la influencia y albergan creencias más radicales (por ejemplo, Baldassari y Bearman 2007; Martins 2008; Salzarulo 2006). Duggins (2017) va aún un paso más allá y asume que a los agentes más radicales no solo les cuesta más cambiar su opinión, sino también falsificarla. Sin embargo, estos son supuestos dudosos que habitualmente se asumen sin mayor justificación. Es cierto que, en ocasiones, se esgrimen teorías como la information or persuasive arguments theory para sostenerlos (por ejemplo, Baldassari y Bearman 2007). En el marco de dicha teoría, Visser, Bizer y Krosnick (2006) muestran evidencia de que, efectivamente, a la gente le cuesta más cambiar de opinión y es más resistente a la influencia cuando, entre otras cosas, está más segura de sus creencias o es más radical. No obstante, dicha evidencia no señala que las personas más seguras de sus creencias sean también más radicales. Por otro lado, en los experimentos de laboratorio con los que Moussaïd et al. (2013) y Chacoma y Zanette (2015) calibran sus modelos de dinámica de opiniones, no se halló prueba alguna de que la seguridad de la veracidad de las creencias y la resistencia a la influencia estén correlacionadas con lo extremo de las mismas ${ }^{9}$. Finalmente, hasta donde nosotros conocemos, no existe evidencia de que la radicalidad de las creencias se correlacione tampoco con la resistencia a falsificarlas.

Algunos modelos contemplan la posibilidad de que las personas con mayor compromiso con sus creencias sean también más influyentes (que gocen de mayor persuasiveness o suportiveness). Por ejemplo, los citados Moussaïd et al. (2013) encuentran en sus experimentos (y después incorporan a su modelo) que un agente puede cambiar su opinión por la de otros agentes cuando esos otros agentes: a) están muy confiados de la bondad de su opinión o b) cuando no están muy confiados, pero son muchos (merced a la heurística de la prueba social).

\section{Fanáticos y moderados}

Muchos modelos incluyen fanáticos (zealots), en ocasiones denominados agentes inflexibles o "tozudos". Se trata de agentes radicales que no cambian de opinión. Por ejemplo, Acemoglu et al. (2013) o Mobilia, Petersen y Redner (2007) presentan variaciones del voter model con este tipo de agentes. En algunos de esos modelos, la presencia de fanáticos está ligada a la existencia de confidence levels heterogéneos. Los fanáticos serían agentes, no solo con una opinión extrema, sino con un confidence level más alto que el del resto de la población. Otros modelos incluyen también moderados, un tipo de agentes que, en función de su número, puede acabar teniendo un rol decisivo en la transición de la polarización al consenso (García-Valdecasas 2013).

\section{HOMOFILIA}

\section{Bounded confidence}

En muchos modelos los agentes están dotados de un cierto grado de homofilia. Los primeros en incorporar esta razonable característica fueron los modelos sociofísicos continuos bounded confidence, aunque ahora prácticamente todos los modelos con una variable de opinión continua (o discreta con más de dos opciones) lo contemplan (por ejemplo, Baldassarri y Bearman 2007; Balenzuela, Pinasco y Semeshenko 2015; Dandekar, Goel y Lee 2013; Duggins 2017; Fortunato 2004, 2005; Gargiulo y Gandica 2017; Kurahashi-Nakamura, Mäs y Lorenz 2016; Sen 2012). Habitualmente, todos los agentes tienen el mismo umbral (confidence level) para dejarse influir por otros, aunque en los últimos tiempos también han aparecido modelos en los que los agentes tienen confidence levels heterogéneos (por ejemplo, Kou et al. 2012; Lorenz 2006, 2010; Mirtabatabaei y Bullo 2012; Weisbuch et al. 2002, 2003).

Influencia social negativa. En los modelos bounded confidence clásicos, cuando dos agentes tienen opiniones muy dispares, más allá de un determinado confidence level, no se influyen mutuamente. En los últimos años, empero, han aparecido modelos que van un paso más allá y contemplan la posibilidad de que, cuando se produce una interacción entre dos agentes con opiniones muy dispares, estos puedan influenciarse negativamente y acabar alejando aún más sus posturas (por ejemplo, Baldassarri y Bearman 2007; Balenzuela, Pinasco y Semeshenko 2015; Duggins 2017; Flache y Mäs 2008; Jager y Amblard 2005; Macy et al. 2003; Mark 2003; Salzarulo 2006).

\section{EL MECANISMO DE CAMBIO DE OPINIÓN}

En todos los modelos existentes la principal (o única) causa próxima del cambio de opinión de los agentes es algún mecanismo de influencia social. En los modelos sociofísicos clásicos, los agentes cambian de opinión, única y exclusivamente, a través de la influencia ejercida por otros agentes; y lo hacen de manera determinista. Además, dicha influencia consiste en una tendencia al conformismo: cuando un agente influye a otro, sus opiniones se aproximan en mayor o menor medida. 
En los últimos años han ido apareciendo modelos que representan variaciones en ambos aspectos. En primer lugar, cada vez hay más modelos no deterministas que introducen azar en el proceso de formación de la opinión (Dandekar, Goel y Lee 2013; KurahashiNakamura, Mäs y Lorenz 2016; Mäs, Flache y Helbing 2010; y para referencias previas a 2009, Castellano, Fortunato y Loreto 2009: 610). Con el recurso al azar, dichos modelos pretenden tomar en consideración otros factores diferentes de la influencia social que puedan estar influyendo en la actualización de las opiniones de los agentes, como la variabilidad en la naturaleza de los distintos individuos o factores externos como los medios de comunicación o la propaganda.

Con todo, a nuestro modo de ver, existe una laguna teórica importante en este punto. No hay modelos de simulación que tomen en consideración, de forma sistemática, el papel del aprendizaje a partir de la propia experiencia en los procesos de formación de la opinión. No obstante, existen buenas razones para pensar que este puede ser un importante mecanismo (Visser, Bizer y Krosnick, 2006; Lohmann 1994: 84). Más aún, la propia experiencia bien puede ser, de hecho, un factor que mitigue el efecto de la influencia de los pares o de los medios de comunicación. Tal y como, por ejemplo, argumentara Kuran (1991b; 2001), las creencias basadas en la propia experiencia son más resistentes a la influencia social. De esta manera, en el ejemplo de Kuran, incluso las personas que vivían en las dictaduras comunistas de la Europa del Este y que tan solo tenían acceso a opiniones favorables al régimen (ya fuesen emitidas por sus conciudadanos o por los medios) podían continuar manteniendo opiniones críticas con el régimen si en su vida cotidiana padecían las colas y la escasez de acceso a los productos básicos, la arbitrariedad de los funcionarios púbicos, la falta de libertad, etc.

Por otro lado, como acaba de mencionarse, en los modelos clásicos la influencia social se asimilaba invariablemente al conformismo. No obstante, muchos modelos actuales incorporan preferencias, lo que Duggins (2017) o Smaldino y Epstein (2015) denominan distinctiveness. La distinctiveness es una forma de influencia social que va en sentido contrario al conformismo: los agentes desean distanciarse de la opinión de los demás. Existe numerosa evidencia empírica de la existencia de este tipo de motivaciones (Imhoff y Erb 2009; Leonardelli, Pickett y Brewer 2010; Maslach, Stapp y Santee 1985; Nail, MacDonald y Levy 2000), de manera que su incorporación a los modelos debe considerarse como un nuevo paso positivo en la dirección del mayor realismo de los mismos. Por otro lado, ni que decir tiene que la idea de la influencia social negativa, presentada al final del apartado anterior, se encuentra claramente relacionada con este mecanismo.

Otros modelos incorporan ideas similares. Por ejemplo, muchos modelos sociofísicos incorpo- ran opositores (contrarians), a saber, agentes que toman la opinión opuesta a la mayoritaria (Galam 2004; Stauffer y Sá Martins 2004). Otros muchos modelos incorporan, por ejemplo, agentes no conformistas o anticonformistas. En el primer caso, el agente mantiene o cambia su opinión al azar, independientemente de la influencia que reciba. En el segundo caso, el agente se comporta como un contrarian con una determinada probabilidad. En este sentido, algunas referencias importantes son Altafini (2013), Buechel, Hellmann y Klößner (2015), Cao et al. (2012), Eger (2013), Galam (2004), Mäs et al. (2013), Mäs, Flache y Kitts (2014), Jarman et al. (2015), Javarone (2014), Kurmyshev y Juárez (2013), Kurmyshev, Juárez y González-Silva (2011), Nyczka, Sznajd-Weron y Cisło (2012) y Regnault, Schabanel y Thierry (2009).

Con todo, cabe insistir en que continúa siendo cierto que, en la totalidad de los modelos, la principal (o única) causa próxima del cambio de opinión de los agentes es algún mecanismo de influencia social. Al fin y al cabo, la distinctiveness, la influencia social negativa o la tendencia a comportarse como un contrarian no dejan de ser formas de influencia social ya que, aunque sea de forma reactiva, la opinión de un agente depende de cuál sea la opinión de otros agentes.

\section{FALSIFICACIÓN DE PREFERENCIAS}

Prácticamente, no existe ningún modelo de simulación que tome en consideración la falsificación de preferencias en el estudio de las dinámicas de opinión pública, siendo Duggins (2017) y Makowsky y Rubin (2013) las únicas excepciones.

Como señala Duggins (2017), seguramente este es uno de los principales, sino el principal déficit de la literatura en este ámbito. La ausencia resulta sorprendente cuando hace ya más de dos décadas que Kuran demostrara con sus modelos (1987a, 1987b, 1989, 1990, 1991a, 1991b) que la falsificación de preferencias puede jugar un papel absolutamente central en las dinámicas de opinión. Sorprendentemente, los trabajos de Kuran tuvieron poca continuidad y tan solo existen un puñado de modelos formales más que incorporan la idea de falsificación de preferencias, no estando estos necesariamente centrados en el estudio de las dinámicas de la opinión pública (Daniel, Arce y Sandler 2003; Bernheim 1994; Ginkel y Smith 1999; Klick y Parisi 2008; Rubin 2014; Tullock 1974).

\section{ELPAPELDELOSMEDIOSDECOMUNICACIÓN ENLAFORMACIÓNDELAOPINIÓNPÚBLICA}

Los trabajos clásicos sobre opinión pública basados en la idea de la aguja hipodérmica explicaban la configuración de la opinión pública como un proceso topdown, por lo que daban a los medios de comu- 
nicación un papel absolutamente fundamental en la conformación de dicha opinión. Curiosamente, empero, los primeros modelos sociofísicos se fueron al extremo contrario, con una aproximación bottomup que obvia el papel de los medios.

No obstante, probablemente la verdad está en algún punto intermedio entre ambas posturas. De este modo, cada vez hay más modelos que incorporan el papel de los medios (Gargiulo y Gandica 2017; Grabowsky y Kosinsky 2006; Kurahashi-Nakamura, Mäs y Lorenz 2016; Watts y Dodds 2007). En la mayoría de modelos la influencia de los medios se incorpora como una modulación exógena. En algunos modelos la opinión va en un único sentido, mientras que en otros se introducen medios rivales ${ }^{10}$. Como en otros casos, desde nuestro punto de vista, ninguna de las dos opciones es "mejor" que la otra per se. Una influencia en un único sentido capturará bien aquellos casos en que todos los medios de comunicación emiten una única opinión (por ejemplo, aunque no solamente, en las dictaduras), mientras que la presencia de distintas influencias en diferentes direcciones será más adecuada en aquellos casos en que exista pluralismo.

Una manera diferente de modelizar el papel de los medios es la de González-Avella et al. (2006, 2007, 2012). En este caso, el modelo consiste en una matriz cuadrada en la que los agentes son influenciados por dos opiniones: la local (el grupo de agentes con los que interactúan), y la global (definida como la opinión modal en el conjunto de la matriz). Los autores sugieren que la opinión global puede entenderse como una influencia cultural transmitida a través de los medios. No obstante, en este modelo, ciertamente los medios de comunicación desempeñan un papel menor, el de meros informadores del estado de la opinión pública.

Finalmente, algunos modelos incorporan también líderes de opinión. Por ejemplo, Chacoma y Zanette (2015) construyen un modelo con agentes más influyentes que el resto y que no son influenciables (en este sentido, serían fanáticos) para tratar de capturar el papel de dichos líderes.

\section{OPINIÓN DE REFERENCIA}

En la gran mayoría de modelos existentes, la dinámica se genera, exclusivamente, en las interacciones a nivel local, por lo que la opinión global del conjunto de la población no afecta directamente a los agentes. Dicho de otro modo, estos no interactúan con el agregado. No obstante, esto parece una limitación, dado que es razonable suponer que la gente tiene alguna noción de cuál es el estado de la opinión pública global (más allá de cuál sea la opinión de la gente con la que ellos interactúen habitualmente en sus redes sociales).
Por motivos obvios, en prácticamente ningún modelo los agentes interactúan exclusivamente con el agregado. Al fin y al cabo, tal y como se ha argumentado más arriba, la principal virtud de un $A B M$ (frente a los modelos matemáticos) es que permite reproducir la emergencia del fenómeno bajo estudio a partir de las interacciones a nivel local ${ }^{11}$. Pero sí que existen algunos modelos en los que los agentes toman en consideración también la opinión global. Los trabajos de González-Avella et al. (2012, 2007, 2006) vuelven a encajar en este punto.

\section{TOPOLOGÍAS}

Tal y como se ha descrito más arriba, en los primeros modelos sociofísicos binarios los agentes estaban ubicados en topologías extremadamente simples. En el voter model y en el Sznajd model los agentes están colocados regularmente en un espacio unidimensional o en una cuadrícula y solo pueden interactuar con sus vecinos adyacentes. En el majority rul, por el contrario, los agentes pueden llegar a interactuar con cualquier otro agente de la población sin ninguna limitación.

Respecto a los sociofísicos continuos bounded confidence, en el Hegselmann-Krause, como se ha visto, los agentes interactúan con un agregado de nivel meso, mientras que en el Deffuant los agentes pueden interactuar con cualquier otro agente dentro de su red.

Hoy en día continúan apareciendo modelos con topologías muy simples. Por ejemplo, en Jarman et al. (2015) se presenta un autómata celular en el que los agentes, que están ubicados en las celdas de un espacio toroidal, son influidos única y exclusivamente por los vecinos de su vecindario de Moore de radio 1 , es decir, por sus ocho vecinos adyacentes. O, también por ejemplo, Martins (2008) implementa su modelo CODA sobre los modelos voter y Sznajd, adoptando, por tanto, sus topologías. No obstante, la tendencia general hoy en día consiste en el desarrollo de modelos con topologías cada vez más sofisticadas, como pueden ser redes de escala libre, redes de mundo pequeño, redes aleatorias, redes completas, redes jerárquicas, etcétera (Baldassarri y Bearman 2007; Battiston et al. 2016; Duggins 2017; Elgazzar 2003; Fortunato 2004, 2005; Fortunato y Stauffer 2006; Gil y Zanette 2006; Grabowsky y Kosinsky 2006; Javarone y Squartini 2015; Sobkowicz 2003, 2010; Sousa 2004, 2005). En realidad, los modelos sociofísicos clásicos pueden entenderse como una regla de actualización de creencias que puede aplicarse a diferentes topologías y contextos, por lo que existen también desarrollos de estos modelos con redes mucho más sofisticadas.

Una cuestión muy ligada a la de la topología es la de cuántos agentes interactúan a la vez en cada ronda de la simulación. En muchos modelos, clásicos y actuales, los agentes interactúan en parejas. 
Este es el caso del voter model o el Deffuant, así como de ciertos modelos actuales como, por ejemplo, Baldassarri y Bearman (2007), Chacoma y Zanette (2015), Laguna et al. (2005) o Moussaïd et al. (2013). En otros modelos, los agentes interactúan en grupos de tamaño y composición variables. Por ejemplo, recuérdese que en el majority rule en cada ronda se forman grupos de $n$ agentes escogidos del conjunto de la población y que el tamaño del grupo varía de una ronda a la otra ${ }^{12}$; en el Sznajd se forman grupos de cuatro vecinos en cada ronda; $y$ en el Hegselmann-Krause los agentes interactúan en cada ronda con todos los otros agentes que quedan dentro de su confidence level. Más allá de los modelos sociofísicos (por ejemplo, Duggins 2017), los agentes interactúan en cada ronda con todos los agentes que forman parte de su red.

Desde nuestro punto de vista, en general, el desarrollo de modelos con estructuras topológicas más complejas ha significado un avance positivo en la dirección de dotar de mayor realismo a los modelos. Por otra parte, el hecho de que los agentes interactúen por parejas o en grupos de un determinado tamaño puede ser pertinente o no, dependiendo del caso concreto que se desee estudiar.

\section{Resultados DE LOS MODELOS}

Siguiendo a Castellano, Fortunato y Loreto (2009: 594), distinguiremos entre tres tipos de modelos en función de los resultados que generan. Los modelos pueden generar consenso (todos los agentes comparten el mismo estado), polarización (hay varios estados posibles, pero solo sobreviven dos) y fragmentación (sobreviven más de dos estados). Según Duggins (2017: 1.4), aun podemos subdividir los modelos que no generan consenso en dos grandes bloques, en función de si generan diversidad débil (las opiniones convergen en un número limitado de estados atractores -polarización o fragmentación-) o diversidad fuerte (las opiniones varían suavemente a lo largo de un espectro continuo).

Como se ha mencionado más arriba, los primeros modelos matemáticos se interesaban por la emergencia del orden y producían consenso. En ese contexto, Abelson formuló su célebre Abelson's diversity puzzle, en el cual demostró que, cuando los agentes se influyen positivamente unos a otros en el marco de una red totalmente conectada, el resultado inevitable es la unanimidad. El problema consiste en que, como argumenta el propio Duggins (2017: 1.4), en el mundo real, totalmente conectado, existe diversidad fuerte.

Por definición, los modelos binarios solo pueden generar consenso o polarización y los discretos (de más de 2 opciones) añaden a esos dos estados la fragmentación. Los modelos existentes binarios y discretos de más de dos opciones pueden generar los tres estados en función de múltiples factores.
Más complicado es el caso de los modelos continuos. En principio, estos modelos deberían poder generar también diversidad fuerte, pero esto no es así en realidad. Los modelos bounded confidence clásicos solo consiguen generar diversidad débil si se dan ciertas condiciones muy restrictivas, cuando el confidence level es muy alto y los agentes nunca son influidos por los diferentes. Cuando el supuesto se relaja, aunque sea poco, los modelos acaban en consenso (Kurahashi-Nakamura, Mäs y Lorenz 2016, Duggins, 2017, Gargiulo y Gandica, 2017: 3.5). En general, hay buenas razones para creer que estos modelos no pueden sostener diversidad fuerte (Duggins, 2017: 1.4., Mäs, Flache y Kitts 2014).

Hoy en día hay una gran variedad de modelos continuos que producen consenso o diversidad débil en función de múltiples factores. Por poner solo algunos ejemplos, Kurahashi-Nakamura, Mäs y Lorenz (2016) son capaces de generar diversidad débil con un bounded confidence con el supuesto de homofilia relajado, introduciendo ruido; mientras que Smaldino y Epstein (2015) generan consenso con un modelo en que los agentes buscan la distinctiveness. Otros modelos han generado diversidad débil a partir de la influencia social negativa (Macy et al. 2003; Mark 2003; Salzarulo 2006; Flache y Mäs 2008), la comunicación de argumentos persuasivos (Mäs et al. 2013; Mäs y Flache 2013), la asimilación sesgada (Dandekar, Goel y Lee 2013), la acumulación de información (Shin y Lorenz 2010), el deseo de la originalidad (Mäs, Flache y Helbing 2010), la atracción por los puntos de vista iniciales (Friedkin 2015) o la influencia de los medios de comunicación o los líderes de opinión (Watts y Dodds 2007). Pero, en cualquier caso, solo existe un modelo con una variable de opinión continua (Duggins 2017) capaz de hacer emerger diversidad fuerte.

\section{CONCLUSIONES:AVANCESYDESAFÍOSENLA MODELIZACIÓNDELADINÁMICADELAOPINIÓN}

La principal aproximación de las ciencias sociales al conocimiento de la opinión pública ha estado tradicionalmente dominada por un interés meramente descriptivo con fines informativos y de asesoramiento. Las escasas aproximaciones causales, como la de la aguja hipodérmica (Lasswell 1927), apuntaban a procesos poco plausibles a nivel cognitivo y desconsideraban el rol de las interacciones sociales. Afortunadamente, como hemos visto en este trabajo, se ha producido en los últimos años un auténtico giro hacia una aproximación causal y generativa, de manera que han comenzado a construirse modelos de vocación explicativa en los que las interacciones a nivel local, microscópico, generan las regularidades que observamos a nivel macroscópico en la opinión pública. 
Como hemos visto, este giro ha estado ampliamente protagonizado por la sociofísica. A pesar de la contribución indiscutible al conocimiento que se ha realizado desde esta disciplina, el dominio del enfoque sociofísico ha supuesto también una proliferación de modelos no validados ni calibrados empíricamente. Los modelos sociofísicos clásicos estaban basados en unos supuestos extremadamente poco realistas. En un origen se importaron al campo de las dinámicas de opinión modelos que se usaban para estudiar fenómenos físicos. Modelos como el voter, por ejemplo, se derivan del modelo Ising, originariamente utilizado en el campo del ferromagnetismo, con unos spins que pueden asumir dos valores $( \pm 1)$. Esta lógica se importó tal cual al estudio de las dinámicas de opinión y simplemente se asumió que el \pm 1 correspondía a estar a favor o en contra del tema de discusión. Por su parte, en el caso de los modelos continuos, se optó por modelizar las dinámicas de opinión como procesos kinéticos (Chacoma y Zanette 2015: 2).

Dichos modelos se basaban, en primer lugar, en topologías y dinámicas extremadamente simples. Por citar solo un par de ejemplos, en el Sznajd model los agentes se encuentran ubicados en un espacio unidimensional e interactúan exclusivamente con sus vecinos adyacentes. Menos realista resulta el Hegselmann-Krause, en el que los agentes interactúan al mismo tiempo con todos los otros agentes de la población que se encuentren dentro de su confidence level (y exclusivamente con ellos). En segundo lugar, y el problema es más grave si cabe aquí, la descripción de las características y procesos cognitivos de los agentes es también extremadamente poco realista. En este sentido, resulta especialmente problemática la falta de soporte empírico en la formalización de la regla de actualización de creencias de los agentes. En efecto, como ha venido argumentándose, la práctica totalidad de los modelos de dinámica de opiniones suponen que la principal (o única) causa del cambio de opinión de los agentes es algún mecanismo de influencia social. No obstante, como señalan Watts y Dodds (2009: 477) "social influence is thus not a singular phenomenon, or even (yet) a well-defined family of phenomena, but rather a blanket label for a loose congregation of social, psychological, and economic mechanisms". En este sentido, en la mayoría de modelos sociofísicos clásicos no queda en absoluto claro cuál es el mecanismo psicológico concreto que trata de replicar la regla de actualización del modelo. Más aún, los autores parecen mostrar un sorprendente desinterés por este crucial problema. En este sentido, Moussaïd et al. (2013: 1) apuntan que "the mechanisms of opinion formation remain poorly understood". Como ya se ha mencionado, en la mayoría de modelos binarios los agentes simplemente "se contagian" de la opinión de sus vecinos al entrar en contacto con ellos. Como se ha visto, dichos agentes no están dotados de memoria, de manera que su opinión va variando de un extremo al otro en cada momento del tiempo sin ningún tipo de limitación. En algunos casos, como en el Sznajd model, la dinámica es realmente extraña. Recuérdese, por ejemplo, que en caso de que los dos agentes que forman la díada no estén de acuerdo, cada uno de ellos le contagia su opinión al vecino del otro. Parece difícil no preguntarse qué proceso real se trata de capturar con semejante procedimiento y, sin embargo, los autores ni siquiera se plantean la pregunta.

El diagnóstico no es mejor en el caso de los modelos continuos. En el DeffuantWeisbuch los agentes interactúan en parejas y convergen en la media de sus opiniones. Obviamente, no existe ninguna evidencia empírica que permita sostener que eso es lo que sucede en las interacciones entre personas reales. Como ya se ha mencionado, el proceso es aún más extraño en el caso del Hegselmann-Krause. $Y$ finalmente, el diagnóstico tampoco es mejor en el caso de los modelos de síntesis tipo CODA: en Martins (2008), los agentes actualizan su opinión a partir de procedimientos bayesianos, cuando es de sobra conocido que dicha regla no describe correctamente los procesos de actualización de creencias de las personas reales.

Obviamente, la simplicidad es una virtud importante de un modelo, pero la falta de realismo de la modelización en este ámbito ha llegado a un punto en que cabe preguntarse, como hace Duggins (2017: 1.2), si los resultados de los modelos habituales "(...) properly inform our understanding of human societies". Tal y como argumenta Elster (2007), el escaso realismo de los modelos encontraría quizá una posible vía de justificación si abandonamos la vocación explicativa y valoramos su buena capacidad predictiva (tal y como sucede en sus aplicaciones al estudio de los fenómenos físicos) y precisamente esta concepción instrumentalista de la teoría es la que parecen defender Castellano, Fortunato y Loreto (2009: 592-593). No obstante, lo cierto es que dicha capacidad predictiva no existe $\mathrm{y}$, como argumentara Sobkowicz (2009: 1.1) hace ya una década en su demanda de "a closer link with reality", los modelos sociofísicos adolecen de una clara "lack of conexión to real life examples", de manera que "modelling has became largely a self-sufficient exercise". Críticas similares pueden encontrarse en Duggins (2017), Balenzuela, Pinasco y Semeshenko (2015), Chacoma y Zanette (2015) o Moussaïd et al. (2013).

Parece, por tanto, obvio que un desafío importante en este campo es el de mejorar la fundamentación y calibración empírica de los modelos. Sobkowicz (2009: 2.2.), de hecho, proponía mejorar el grado de realismo en dos campos: el de las redes en que se ubican los agentes del modelo y el de los agentes, especialmente en cuanto a sus procesos cognitivos de cambio de opinión. Como se ha visto en este trabajo, en el terreno de las redes sí se han producido avances significativos en los últimos años. Los progresos, sin embargo, han sido menores en lo que se refiere a la descripción de los 
agentes. Algunos modelos han introducido agentes con diferentes características, más allá de su opinión, como una cierta capacidad de influencia o de resistencia a la influencia, lo que sin duda ha supuesto un avance positivo y ha abierto la puerta a asignar roles y poderes diferenciados a los agentes. A esta mejora "intuitiva" del realismo de los agentes ha de añadirse el ejercicio explícito de construir evidencias empíricas con las que fundamentar el diseño de los agentes. Por ejemplo, recientemente han venido apareciendo modelos calibrados a partir de experimentos de laboratorio realizados previamente, algunos de los cuales han servido para poner en cuestión ciertos supuestos habituales de los modelos sociofísicos. Dos referencias muy notables en este sentido serían Chacoma y Zanettte (2015) y Moussaïd et al. (2013) ${ }^{13}$. A nuestro entender, esta es una práctica aún minoritaria, pero que está destinada a volverse dominante si lo que queremos es construir modelos genuinamente explicativos.

A los dos campos de mejora señalados por Sobkowicz debería añadirse un tercero: el testado empírico de los resultados que producen los modelos. No se trata solo de fundamentar empíricamente el diseño del modelo, sino también de contrastarlo analizando si los patrones macroscópicos que genera reproducen o no los patrones naturales que querríamos explicar. Este problema es, de hecho, reconocido por los propios Castellano, Fortunato y Loreto (2009: 593). A modo de ejemplo, cabe destacar la aportación de Duggins (2017), que aplica su modelo a los datos provenientes de dos bases de datos de opiniones políticas en Estados Unidos y consigue reproducir sus resultados. En este sentido, Duggins muestra cómo cada elemento que introduce en su modelo, y que aumenta la complejidad del mismo, también contribuye significativamente a generar nuevos resultados observados en los casos reales que los modelos anteriores no eran capaces de generar.

Hasta ahora, como hemos dicho, el giro hacia una explicación generativa de la opinión pública ha estado protagonizado por la sociofísica. Sin embargo, es destacable el hecho de que los principales aportes en la mejora del realismo de los modelos están proviniendo de fuera del mundo sociofísico. Esto, sin duda, marca una necesaria línea de trabajo y desarrollo: aquellos científicos sociales que comparten con la sociofísica la idea de que explicar un patrón macroscópico implica demostrar su generación a partir de interacciones microscópicas, tienen en el estudio de la dinámica de la opinión un terreno en el que realizar una aportación imprescindible. Resulta crucial que seamos capaces de aprovechar el impulso generado por la riqueza y la fertilidad de los modelos sociofísicos. Apoyándonos en estos modelos, nuestra contribución debería ser la de ir más allá de ellos, resolviendo más eficazmente los problemas de verificación y validez, construyendo modelos más completos, más realistas, más contrastados empíricamente en sus resultados y, por todo ello, en definitiva, más explicativos.

\section{Agradecimientos}

El presente trabajo se ha beneficiado de la concesión de un proyecto del Ministerio de Economía y Competitividad (MINECO), en el marco del Plan Nacional de I+D+i (ref. CSO2015-64740-R.), así como de un proyecto de la Escola d'Administració Pública de Catalunya de la Generalitat de Catalunya (ref. 2018 EAPC000014).

\section{NOTAS}

[1] Castellano, Fortunato y Loreto se refieren al conjunto de la literatura sociofísica. No obstante, resulta pertinente mantener el adjetivo de "abrumadora" para referirse a la cantidad de literatura producida en este ámbito específico. Por un lado, los modelos de dinámicas de opinión constituyen la principal puerta de entrada de la sociofísica en las ciencias sociales, mientras que, por el otro, los sociofísicos no son ni mucho menos los únicos modelos de dinámicas de opinión.

[2] En las citadas referencias puede hallarse una discusión detallada acerca de en qué consisten las propiedades emergentes de los fenómenos sociales, así como del tipo de modelos que pueden emplearse para su estudio.

[3] Ni que decir tiene que en esta categoría entrarían muchos de los modelos de falsificación de preferencias (hoy ya clásicos) de Timur Kuran (1987a, 1987b, 1989, 1990, 1991a, 1991b).

[4] Los primeros modelos fueron autómatas celulares, aunque en los últimos años se han ido imponiendo, prácticamente en exclusiva, los modelos multiagente. Ver Gilbert y Troitzsch (2005) para una introducción a este tipo de modelos. Véase también Hedström y Manzo (2015) para una discusión en profundidad de sus potencialidades, así como de los principales desafíos a que se enfrentan.

[5] Muchos de los modelos sociofísicos clásicos son tan simples que pueden ser resueltos tanto analítica como numéricamente mediante simulaciones. Para discusiones detalladas de esta cuestión pueden consultarse las revisones de la literatura citadas más arriba.

[6] Todos estos modelos han sido fuertemente influenciados por el célebre Ising model, que también ha tenido su aplicación al estudio de las dinámicas de opinión. Ver Galam, Gefen, Shapir (1982) y Galam y Moscovici (1991).

[7] Lo que sigue se basa en Castellano, Fortunato y Loreto (2009), donde puede hallarse una descripción más detallada de estos, así como de algunos otros modelos clásicos que no se presentan aquí.

[8] Ciertamente, en los continuos también estaban dotados de un confidence level. Pero el problema en este punto es el mismo.

[9] No obstante, cabe mencionar que en ambos casos los experimentos se realizan con preguntas sobre cuestiones fácticas, poco controvertidas y que cabría la posibilidad de que los resultados cambiasen si se utilizasen temas "calientes" o controvertidos. 
[10] Como se ha mencionado anteriormente, algunos modelos introducen azar con la finalidad de capturar cosas como el papel de los medios de comunicación.

[11] No obstante, Smaldino y Epstein (2015) constituyen una interesante excepción en este punto.

\section{REFERENCIAS BibLIOGRÁfiCAS}

Abelson, R. P. 1964. "Mathematical Models of the Distribution of Attitudes Under Controversy". Pp. 142-160 en Contributions to Mathematical Psychology, editado por N. Frederiksen y H. Gulliksen. New York: Holt, Rinehart, and Winston.

Acemoğlu, D., G. Como, F. Fagnani y A. Ozdaglar. 2013. "Opinion fluctuations and disagreement in social networks", Mathematics of Operations Research 38(1): 127. https://doi.org/10.1287/moor.1120.0570

Altafini, C. 2013. "Consensus problems on networks with antagonistic interactions", IEEE Transactions on Automatic Control 58(4): 935-946. https://doi.org/10.1109/ TAC.2012.2224251

Axelrod, R. 1997. "The dissemination of culture: A model with local convergence and global polarization", Journal of conflict resolution 41(2): 203-226

Baker, G. A. y J. P. Hague. 2008. "Rise of the centrist: from binary to continuous opinion dynamics", International Journal of Modern Physics C 19(09): 14591475. https://doi.org/10.1142/S0129183108013023

Baldassarri, D. y P. Bearman. 2007. "Dynamics of political polarization", American Sociological Review 72(5): 784811. https://doi.org/10.1177/000312240707200507

Balenzuela P., J.P. Pinasco y V. Semeshenko. 2015. "The Undecided Have the Key: Interaction-Driven Opinion Dynamics in a Three State Model", PLoS ONE 10(10): e0139572. https://doi.org/10.1371/journal.pone.0139572

Bartolozzi, M., D. B. Leinweber y A. W. Thomas. 2005. "Stochastic opinion formation in scale-free networks", Physical Review E 72: 046113 https://doi.org/10.1103/ PhysRevE.72.046113

Barth, V. 2006. "A model of opinion dynamics among firms", en Potentials of Complexity Science for Business, Government, and the Media, editado por D. Helbing. Budapest: Collegium Budapest

Battiston, F., A. Cairoli, V. Nicosia, A. Baule y V. Latora. 2016. "Interplay between consensus and coherence in a model of interacting opinions", Physica D: Nonlinear Phenomena, 323: 12-19. https://doi.org/10.1016/j. physd.2015.10.013

Behera, L. y F. Schweitzer. 2003. "On Spatial Consensus Formation: Is the Sznajd Model Different from a Voter Model?", International Journal of Modern Physics C 14(10): 1331-1354. https://doi.org/10.1142/ S0129183103005467

Bernardes, A.T., U.M.S. Costa, A.D. Araujo y D. Stauffer. 2001. Damage spreading, coarsening dynamics and distribution of political votes in Sznajd model on square lattice. International Journal of Modern Physics C, 12(02), pp.159-167.

Bernardes, A.T., D. Stauffer y J. Kertész. 2002. Election results and the Sznajd model on Barabasi network. The European Physical Journal B-Condensed Matter and Complex Systems, 25(1), pp.123-127.

Bernheim, B.D. 1994. "A Theory of Conformity", Journal of Political Economy 102(5): 841-877. https://doi. org/10.1086/261957

Berger, R.L. 1981. "A necessary and sufficient condition for reaching a consensus using DeGroot's method", Journal of the American Statistical Association 76(374): 415418. https://doi.org/10.1080/01621459.1981.10477662
[12] En una variante (Tessone et al. 2004) se forman grupos de tamaño diverso en una misma ronda.

[13] Ver también Lorenz et al. (2011) y Mavrodiev, Tessone y Schweitzer (2013).

Buechel, B., T. Hellmann y S. Klößner. 2015. "Opinion dynamics and wisdom under conformity", Journal of Economic Dynamics and Control 52: 240-257. https://doi. org/10.1016/j.jedc.2014.12.006

Cao, Z., M. Yang, X. Qu y X. Yang. 2012. "Rebels lead to the doctrine of the mean: opinion dynamic in a heterogeneous DeGroot model", arXiv preprint arXiv:1204.0161. https://arxiv.org/abs/1204.0161

Castellano, C., S. Fortunato y V. Loreto. 2009. "Statistical physics of social dynamics", Reviews of modern physics, 81(2): 591-646. https://doi.org/10.1103/RevModPhys.81.591

Chacoma, A. y D. H. Zanette. 2015. "Opinion Formation by Social Influence: From Experiments to Modeling", PLoS ONE 10(10): e0140406. https://doi.org/10.1371/journal.pone.0140406

Clifford, P. y A. Sudbury. 1973. "A model for spatial conflict", Biometrika 60(3): 581588. https://doi.org/10.1093/biomet/60.3.581

Crokidakis, N. y P. M. C. De Oliveira. 2011. "The Sznajd model with limited persuasion: competition between highreputation and hesitant agents", Journal of Statistical Mechanics: Theory and Experiment, 2011(11): P11004. https://doi.org/10.1088/1742-5468/2011/11/P11004

Dall'Asta, L. y C. Castellano. 2007. "Effective surface-tension in the noise-reduced voter model", EPL (Europhysics Letters) 77(6): 60005. https://doi.org/10.1209/02955075/77/60005

Dandekar, P., A. Goel y D. T. Lee. 2013. "Biased assimilation, homophily, and the dynamics of polarization", Proceedings of the National Academy of Sciences 110(15): 5791-5796. https://doi.org/10.1073/pnas.1217220110

Daniel, G., M. Arce y T. Sandler. 2003. "An evolutionary game approach to fundamentalism and conflict", Journal of Institutional and Theoretical Economics JITE 159(1): 132-154. https://doi.org/10.1628/0932456032975096

Deffuant, G., D. Neau, F. Amblard y G. Weisbuch. 2000. "Mixing beliefs among interacting agents", Advances in Complex Systems 3(01n04): 87-98. https://doi. org/10.1142/S0219525900000078

Duggins, P. 2017. "A psychologically-motivated model of opinion change with applications to American politics", Journal of Artificial Societies and Social Simulation 20(1): 13. https://doi.org/10.18564/jasss.3316

Eger, S. 2013. "Opinion dynamics under opposition", arXiv preprint arXiv:1306.3134. https://doi.org/10.2139/ ssrn.2352555

Elgazzar, A. S. 2003. "Applications of small-world networks to some socio-economic systems", Physica A: Statistical Mechanics and its Applications 324(1-2): 402407. https://doi.org/10.1016/S0378-4371(02)01956-8

Elster, J. 2007. Explaining social behavior: More nuts and bolts for the social sciences. Cambridge: Cambridge University Press.

Flache, A. y M. Mäs. 2008. "How to get the timing right. A computational model of the effects of the timing of contacts on team cohesion in demographically diverse teams", Computational and Mathematical Organization Theory 14(1): 23-51. https://doi.org/10.1007/s10588-008-9019-1 
Flache, A., M. Mäs, T. Feliciani, E. Chattoe-Brown, G. Deffuant, S. Huet y J. Lorenz. 2017. "Models of Social Influence: Towards the Next Frontiers", Journal of Artificial Societies \& Social Simulation, 20(4).

Fortunato, S. 2004. "Universality of the threshold for complete consensus for the opinion dynamics of Defuant et al", International Journal of Modern Physic C 15(09): 13011307. https://doi.org/10.1142/S0129183104006728

Fortunato, S. 2005. "The Sznajd consensus model with continuous opinions", International Journal of Modern Physics C 16(01): 17-24. https://doi.org/10.1142/ S0129183105006917

Fortunato, S., V. Latora, A. Pluchino y A. Rapisarda. 2005. "Vector opinion dynamics in a bounded confidence consensus model", Interational Journal of Modern Physics C 16(10): 1535-1551. https://doi.org/10.1142/ S0129183105008126

Fortunato, S. y D. Stauffer. 2006. "Computer simulations of opinions and their reactions to extreme events". Pp. 233-257 en Extreme events in nature and society, editado por Sergio Albeverio, S., Jentsch, V. y H. Kantz. Berlin, Heidelberg: Springer.

French, J. R. P. Jr. 1956. "A formal theory of social power", Psychological Review 63(3): 181-194. http:/l dx.doi.org/10.1037/h0046123

Friedkin, N.E. 2015. "The problem of social control and coordination of complex systems in sociology: A look at the community cleavage problem", IEEE Control Systems 35(3): 40-51. https://doi.org/10.1109/ MCS.2015.2406655

Galam, S. 2002. "Minority opinion spreading in random geometry", The European Physical Journal B, 25(4):403406. https://doi.org/10.1140/epjb/e20020045

Galam, S. 2004. "Contrarian deterministic effects on opinion dynamics: 'the hung elections scenario"'. Physica A 333: 453-460. https://doi.org/10.1016/j.physa.2003.10.041

Galam, S. 2008. "Sociophysics: A review of Galam models", International Journal of Modern Physics C 19(03): 409440. https://doi.org/10.1142/S0129183108012297

Galam, S., Y. Gefen, e Y. Shapir. 1982. "Sociophysics: A new approach of sociological collective behaviour. I. meanbehaviour description of a strike", Journal of Mathematical Sociology 9(1): 1-13. https://doi.org/10. 1080/0022250X.1982.9989929

Galam, S. y S. Moscovici. 1991. "Towards a theory of collective phenomena: Consensus and attitude changes in groups", European Journal of Social Psychology 21(1): 49-74. https://doi.org/10.1002/ejsp.2420210105

Galam, S. y M. A. Javarone. 2016. "Modeling radicalization phenomena in heterogeneous populations", PLOS ONE 11(5): e0155407. https://doi.org/10.1371/journal. pone. 0155407

García-Valdecasas, J. I. 2013. "Grupos moderados y tolerantes en la polarización política de las redes sociales", Revista Española de Investigaciones Sociológicas 142(1): 25-44.

Gargiulo, F. e Y. Gandica. 2017. "The role of homophily in the emergence of opinion controversies", Journal of Artificial Societies and Social Simulation 20(3):8. https:// doi.org/10.18564/jasss.3448

Gekle, S., L. Peliti y S. Galam. 2005. "Opinion dynamics in a three-choice system", The European Physical Journal $B$ - Condensed Matter and Complex Systems B 45: 569-575. https://doi.org/10.1140/epjb/e2005-00215-3

Gil, S. y D. H. Zanette. 2006. "Coevolution of agents and networks: Opinion spreading and community discon- nection", Physics Letters A 356(2): 89-94. https://doi. org/10.1016/j.physleta.2006.03.037

Gilbert, N. Troitzsch, K. 2005. Simulation for the social scientist. McGraw-Hill Education (UK).

Ginkel, J. y A. Smith. 1999. "So you say you want a revolution: A game theoretic explanation of revolution in repressive regimes", Journal of Conflict Resolution 43(3): 291316. https://doi.org/10.1177/0022002799043003002

González-Avella, J. C., Eguíluz, V. M., Cosenza, M. G., Klemm, K., J. Herrera y M. San Miguel. 2006. "Local versus global interactions in nonequilibrium transitions: A model of social dynamics", Physical Review E 73(4): 046119. https://doi.org/10.1103/PhysRevE.73.046119

González-Avella, J. C., Cosenza, M. G., Klemm, K., V.M. Eguíluz y M. San Miguel. 2007. "Information feedback and mass media effects in cultural dynamics", Journal of Artificial Societies and Social Simulation 10(39)1.

González-Avella, J. C., M.G. Cosenza y M. San Miguel. 2012. "A model for cross-cultural reciprocal interactions through mass media", PloSone 7(12): e51035. https:// doi.org/10.1371/journal.pone.0051035

Grabowsky, A. y R. A. Kosinsky. 2006. "Ising-based model of opinion formation in a complex network of interpersonal interactions", Physica A 361: 651-664. https://doi. org/10.1016/j.physa.2005.06.102

Groot, M.H. de 1974. "Reaching consensus", Journal of American Statistical Association 69: 118-121. https://doi.or g/10.1080/01621459.1974.10480137

Halu, A., K. Zhao, A. Baronchelli y G. Bianconi. 2013. "Connect and win: The role of social networks in political elections", EPL (Europhysics Letters) 102(1): 16002. https://doi.org/10.1209/0295-5075/102/16002

Hedström, P. 2005. Dissecting the Social: On the Principles of Analytical Sociology. Cambridge: Cambridge University Press.

Hedström, P. 2006. "Explaining social change: An analytical approach", Papers. Revista de Sociologia 80: 73-95.

Hedström, P. y P. Bearman (eds.). 2009. The Oxford handbook of analytical sociology. Oxford: Oxford University Press.

Hedström, P. y G. Manzo, (eds.) 2015. "Special Issue: AgentBased Modeling: Advances and Challenges", Sociological Methods \& Research 44(2).

Hegselmann, R. y U. Krause. 2002. "Opinion dynamics and bounded confidence. Models, analysis and simulation", Journal of Artificial Societies and Social Simulation, 5(3):2.

Holley, R. y T. M. Liggett. 1975. "Ergodic theorems for weakly interacting systems and the voter model", The annals of probability 3(4): 643-663.

Hołyst, J. A., K. Kacperski y F. Schweitzer. 2001. "Social impact models of opinion dynamics", Annual Reviews Of Computational Physics IX: 253-273. https://doi. org/10.1142/9789812811578_0005

Imhoff, R. y H. P. Erb. 2009. "What motivates nonconformity? Uniqueness seeking blocks majority influence", Personality and Social Psychology Bulletin 35(3): 309320. https://doi.org/10.1177/0146167208328166

Jager, W. y F. Amblard. 2005. "Uniformity, bipolarization and pluriformity captured as generic stylized behaviour with an agent-based simulation model of attitude change", Computational \& Mathematical Organization Theory 10(4): 295303. https://doi.org/10.1007/ s10588-005-6282-2

Jarman, M., A. Nowak, W. Borkowski, D. Serfass, A. Wong y R. Vallacher. 2015. "The critical few: Anticonformists at the crossroads of minority opinion survival and collap- 
se", Journal of Artificial Societies and Social Simulation, 18(1)6. https://doi.org/10.18564/jasss.2663

Javarone, M. A. 2014. "Social influences in opinion dynamics: the role of conformity", Physica A 414: 19-30. https:// doi.org/10.1016/j.physa.2014.07.018

Javarone M. A. y T. Squartini. 2015. "Conformism-driven phases of opinion formation on heterogeneous networks: the q-voter model case", Journal of Statistical Mechanics: Theory and Experiment P10002. https://doi. org/10.1088/17425468/2015/10/P10002

Jiang, L. L., D.Y. Hua y T. Chen. (2007). "Nonequilibrium phase transitions in a model with social influence of inflexible units". J. Phys. A: Math. Theor. 40: 11271-11276.

Jiang, L. L., D.Y. Hua, J.F. Zhu, B.H. Wang y T. Zhou. 2008. "Opinion dynamics on directed small-world networks", The European Physical Journal B 65(2), 251255. https://doi.org/10.1140/epjb/e2008-00342-3

Klick, J. y F. Parisi. 2008. "Social networks, self-denial, and median preferences", The Journal of Socio-Economics, 37: 13191327. https://doi.org/10.1016/j.socec.2007.08.008

Kou, G., Y. Zhao, Y. Peng e Y. Shi. 2012. "Multi-level opinion dynamics under bonded confidence", PlosOne, 7(9): e43507. https://doi.org/10.1371/journal.pone.0043507

Krause, U. 2000. "A discrete nonlinear and non-autonomous model of consensus formation". Pp. 227-236 en Communications in Difference Equations, editado por Elyadi, S., Ladas, G., Popenda, J. y J. Rakowski, Amsterdam: Gordon and Breach Pub.

Kurahashi-Nakamura, T., M. Mäs y J. Lorenz. 2016. "Robust clustering in generalized bounded confidence models", Journal of Artificial Societies and Social Simulation, 19(4)7. https://doi.org/10.18564/jasss.3220

Kuran, T. 1987a. "Chameleon voters and public choice", Public Choice 53(1): 53-78. https://doi.org/10.1007/ BF00115654

Kuran, T. 1987b. "Preference falsification, policy continuity and collective conservatism", The Economic Journal, 97(387): 642-665. http://www.jstor.org/stable/2232928

Kuran, T. 1988. "The tenacious past: Theories of personal and collective conservatism", Journal of Economic Behavior \& Organization 10(2): 143-171. https://doi. org/10.1016/0167-2681(88)90043-1

Kuran, T. 1989. "Sparks and prairie fires: A theory of unanticipated political revolution", Public Choice 61(1): 41-74. https://doi.org/10.1007/BF00116762

Kuran, T. 1990. "Private and public preferences", Economics and Philosophy 6: 1-26. https://doi.org/10.1017/ S026626710000064X

Kuran, T. 1991a. "Cognitive limitations and preference evolution", Journal of Institutional and Theoretical Economics 147: 241-273. http://www.jstor.org/stable/40751404

Kuran, T. 1991b. "Now out of never: The element of surprise in the East European revolution of 1989", World politics 44(01): 748. https://doi.org/10.2307/2010422

Kuran, T. 1993. "The Unthinkable and the Unthought", Rationality and Society 5(4): 473-505. https://doi. org/10.1177/1043463193005004005

Kuran, T. 1995. Private truths, public lies: the social consequences of preference falsification. Cambridge, Mass: Harvard University Press.

Kuran, T. 2001. "Public opinion, microsociological aspects". Pp. 12556-12560 en International Encyclopedia of the Social and Behavioral Sciences. Amsterdam: Pergamon.

Kurmyshev, E., y H. A. Juárez. 2013. "What is a leader of opinion formation in bounded confidence models?", arXiv preprint arXiv:1305.4677. https://arxiv.org/abs/1305.4677 1-18.
Kurmyshev, E., H.A. Juárez y R.A. González-Silva. 2011. Dynamics of bounded confidence opinion in heterogeneous social networks: Concord against partial antagonism. Physica A: Statistical Mechanics and its Applications, 390(16), pp.2945-2955.

Laguna, M. F., S.R. Gusman, G. Abramson, S. Gonçalves y J. R. Iglesias. 2005. "The dynamics of opinion in hierarchical organizations", Physica A: Statistical Mechanics and its Applications 351(2-4): 580-592. https://doi. org/10.1016/j.physa.2004.11.064

Lallouache, M., A. Chakraborti y B. K. Chakrabarti. 2010a. "Kinetic exchange models for social opinion formation", Science and Culture 76(9-10): 485-488.

Lallouache, M., A.S. Chakrabarti, A. Chakraborti y B.K. Chakrabarti. 2010b. Opinion formation in kinetic exchange models: Spontaneous symmetry-breaking transition. Physical Review E, 82(5), p.056112. https:// doi.org/10.1103/PhysRevE.82.056112

Lasswell, H. 1927. Propaganda techniques in the World War. Cambridge: MIT Press.

Latané, B. 1981. "The psychology of social impact", American Psychologist 36(4): 343356. http://dx.doi. org/10.1037/0003-066X.36.4.343

Leonardelli, G. J., C.L. Pickett y M. B. Brewer. 2010. "Optimal distinctiveness theory: A framework for social identity, social cognition, and intergroup relations", Advances in Experimental Social Psychology 43: 63113. https://doi. org/10.1016/S0065-2601(10)43002-6

Linares, F. 2018. Sociología y teoría social analíticas. Madrid: Alianza editorial.

Lohmann, S. 1994. "The dynamics of informational cascades", World Politics 47: 42101. https://doi. org/10.2307/2950679

Lorenz, J. 2006. "Consensus strikes back in the HegselmannKrause model of continuous opinion dynamics under bounded confidence", Journal of Artificial Societies and Social Simulation 9:8.

Lorenz, J. 2007. "Continuous opinion dynamics under bounded confidence: a survey", International Journal of Modern Physics C 18(12): 1819-1838. https://doi.org/10.1142/ S0129183107011789

Lorenz, J. 2010. "Heterogeneous bounds of confidence: Meet, discuss and find consensus!", Complexity 15(4): 4352. https://doi.org/10.1002/cplx.20295

Lorenz, J., H. Rauhut, F. Schweitzer y D. Helbing. 2011. "How social influence can undermine the wisdom of crowd effect", Proceedings of the National Academy of Sciences 108: 90209025. https://doi.org/10.1073/ pnas. 1008636108

Macy, M. W., J.A. Kitts, A. Flache y S. Benard. 2003. "Polarization in dynamic networks: A hopfield model of emergent structure". Pp. 162173 en Dynamic Social Network Modeling and Analysis. Washington D. C.: National Academies Press.

Makowsky, M. D. y J. Rubin. 2013. "An agent-based model of centralized institutions, social network technology, and revolution", PLoS ONE 8(11): e80380. https://doi. org/10.1371/journal.pone.0080380

Mark, N.P. 2003. "Culture and competition: Homophily and distancing explanations for cultural niches", American Sociological Review 68(3): 319-345. https://doi. org/10.2307/1519727

Marsden, P. V. 1981. "Introducing influence processes into a system of collective decision", American Journal of Sociology 86: 1203-35. https://doi. org $/ 10.1086 / 227383$ 
Martins, A. C. 2008. "Continuous opinions and discrete actions in opinions dynamics problems", International Journal of Modern Physics C 19(4): 617-624. https://doi. org/10.1142/S0129183108012339

Mäs, M., A. Flache y J. A. Kitts. 2014. "Cultural integration and differentiation in groups and organizations". Pp. 71-90 en Perspectives on Culture and Agent-Based Simulations. Nueva York: Springer.

Mäs, M., A. Flache y D. Helbing. 2010. "Individualization as driving force of clustering phenomena in humans", PLoS Computational Biology. 6(10): e1000959. https://doi. org/10.1371/journal.pcbi.1000959

Mäs, M. y A. Flache. 2013. "Diferentiation without distancing. explaining bipolarization of opinions without negative influence", PLOS ONE, 8(11): e74516. https://doi. org/10.1371/journal.pone.0074516

Mäs, M., A. Flache, K. Takacs y K. A. Jehn. 2013. "In the short term we divide, in the long term we unite: Demographic crisscrossing and the effects of fault lines on subgroup polarization", Organization science 24(3): 716-736.

Maslach, C., J. Stapp y R. T. Santee. 1985. "Individuation: Conceptual analysis and assessment", Journal of Personality and Social Psychology 49(3): 729-738. https:// doi.org/10.1037/0022-3514.49.3.729

Mavrodiev, P., C.J. Tessone y F. Schweitzer. 2013. "Quantifying the effects of social influence", Scientific Reports 3: 1360. https://doi.org/10.1038/srep01360

Mirtabatabaei, A. y F. Bullo. 2012. "Opinion dynamics in heterogeneous networks: convergence conjectures and theorems", SIAM Journal on Control and Optimization, 50(5): 2763-2785. https://doi.org/10.1137/11082751X

Mobilia, M., 2011. Fixation and polarization in a three-species opinion dynamics model. EPL (Europhysics Letters), 95(5), p.50002. https://doi.org/10.1209/02955075/95/50002

Mobilia, M., A. Petersen y S. Redner. 2007. "On the role of zealotry in the voter model", Journal of Statistical Mechanics: Theory and Experiment 2007(08): P08029. https://doi.org/10.1088/1742-5468/2007/08/P08029

Moussaïd, M., J.E. Kämmer, P.P. Analytis y H. Neth. 2013. "Social influence and the collective dynamics of opinion formation", Plos One 8(11): e78433. https://doi. org/10.1371/journal.pone.0078433

Nail, P. R., G. MacDonald y D. A. Levy. 2000. "Proposal of a fourdimensional model of social response", Psychological Bulletin 126(3): 454-470. https://doi.org/10.1037/00332909.126.3.454

Nowak, A. y M. Lewenstein. 1996. "Modeling Social Change with Cellular Automata". Pp. 249-285 en Modelling and Simulation in the Social Sciences From A Philosophy of Science Point of View editado por Hegselmann, R., Mueller, U. y K. G. Troitzsch. Dordrecht: Kluver.

Nowak, A., J. Szamrej y B. Latané. 1990. "From private attitude to public opinion: A dynamic theory of social impact", Psychological Review 97(3), 362-376.

Nyczka, P., K. Sznajd-Weron y J. Cisło. 2012. "Phase transitions in the q-voter model with two types of stochastic driving", Physical Review E 86(1): 011105. https://doi. org/10.1103/PhysRevE.86.011105

Regnault, D., N. Schabanel y É. Thierry. 2009. "Progresses in the analysis of stochastic 2D cellular automata: A study of asynchronous 2D minority", Theoretical Computer Science 410(47-49): 4844-4855. https://doi. org/10.1016/j.tcs.2009.06.024

Rubin, J. 2014. "Centralized institutions and cascades", Journal of Comparative Economics 42(2): 340-357. https:// doi.org/10.1016/j.jce.2013.11.002
Sabatelli, L. y P. Richmond. 2003. "Phase transitions, memory and frustration in a Sznajd-like model with synchronous updating", International Journal of Modern Physics C, 14(09), 1223-1229. https://doi.org/10.1142/S0129183103005352

Sabatelli, L. y P. Richmond. 2004. "Non-monotonic spontaneous magnetization in a Sznajd-like consensus model", Physica A: Statistical Mechanics and its Applications, 334(1-2): 274-280. https://doi.org/10.1016/j. physa.2003.10.062

Salzarulo, L. 2006. "A continuous opinion dynamics model based on the principle of meta-contrast", Journal of Artificial Societies and Social Simulation 9(1).

Schulze, C. 2004. "Sznajd opinion dynamics with global and local neighborhood", International Journal of Modern Physics C 15(06): 867-872. https://doi.org/10.1142/ S0129183104006273

Sen, P. 2011. "Phase transitions in a two-parameter model of opinion dynamics with random kinetic exchanges", Physical Review E 83(1): 016108. https://doi. org/10.1103/PhysRevE.83.016108

Sen, P. 2012. "Nonconservative kinetic exchange model of opinion dynamics with randomness and bounded confidence", Physical Review E 86(1): 016115. https://doi. org/10.1103/PhysRevE.86.016115

Shin, J.K. y J. Lorenz. 2010. "Tipping diffusivity in information accumulation systems: More links, less consensus", Journal of Statistical Mechanics: Theory and Experiment 2010(06): P06005. https://doi.org/10.1088/17425468/2010/06/P06005

Sîrbu, A., V. Loreto, V.D.P. Servedio y F. Tria. 2007. "Opinion dynamics: model, extensions and external effects". Pp. 363-401 en Participatory Sensing, Opinions and Collective Awareness. Suiza: Springer, Cham.

Smaldino, P. E. y J. M. Epstein. 2015. "Social conformity despite individual preferences for distinctiveness", Royal Society Open Science 2(3): 140437. https://doi. org/10.1098/rsos.140437

Sobkowicz, P. 2003. "Opinion formation in networked societies with strong leaders", arXiv preprint arXiv:condmat/0311521. https://arxiv.org/abs/cond-mat/0311521

Sobkowicz, P. 2009. "Modeling opinion formation with physics tools: a call for closer link with reality", Journal of Artificial Societies and Social Simulation 12(1): 11.

Sobkowicz, P. 2010. "Effect of leader's strategy on opinion formation in networked societies with local interactions", International Journal of Modern Physics C 21(06): 839852. https://doi.org/10.1142/S0129183110015518

Sousa, A.O. 2004. "Bounded confidence model on a still growing scale-free network", arXiv preprint arXiv:condmat/0406766. https://arxiv.org/abs/cond-mat/0406766

Sousa, A. O. 2005. "Consensus formation on a triad scalefree network", Physica A: Statistical Mechanics and its Applications 348: 701-710. https://doi.org/10.1016/j. physa.2004.09.027

Stauffer, D. 2002. "The Sznajd model of consensus building with limited persuasion", International Journal of Modern Physics C 13(3): 315-317. https://doi.org/10.1142/ S0129183102003140

Stauffer, D. 2003. "How to convince others: Monte Carlo simulations of the Sznajd model". Pp. 147-155 en The Monte Carlo Method in the Physical Sciences, editado por J. E. Gubernatis. Los Alamos: American Institute of Physics.

Stauffer, D. y J. S. Martins. 2004. "Simulation of Galam's contrarian opinions on percolative lattices", Physica A: Statistical Mechanics and its Applications, 334(3-4): 558-565. https://doi.org/10.1016/j.physa.2003.12.003 
Stauffer, D., A. Sousa y C. Schulz. 2004. "Discretized opinion dynamics of the Deffuant model on scale-free networks", Journal of Artificial Societies and Social Simulation 7(3).

Sznajd-Weron, K. (2005). "Sznajd model and its applications", Acta Physica Polonica B 36(8): 2537-2547.

Sznajd-Weron, K. y J. Sznajd. 2000. "Opinion Evolution in a Closed Community", International Journal of Modern Physics C 11(6): 1157-1165. https://doi.org/10.1142/ S0129183100000936

Tessone, C. J., R. Toral, P. Amengual, H.S. Wio y M. San Miguel. 2004. "Neighborhood models of minority opinion spreading", The European Physical Journal B-Condensed Matter and Complex Systems 39(4): 535-544. https://doi.org/10.1140/epjb/e2004-00227-5

Tullock, G. 1974. The social dilemma: The economics of war and revolution. Blacksburg, VA: University Publications.

Vázquez, F., P.L. Krapivsky y S. Redner. 2003. "Constrained opinion dynamics: freezing and slow evolution", Journal of Physics A: Mathematical and General 36(3): 6168. https://doi.org/10.1088/0305-4470/36/3/103
Visser, P. S., G.Y. Bizer y J. A. Krosnick. 2006. "Exploring the latent structure of strength-related attitude attributes." Pp. 1-60 en Advances in experimental social psychology (Vol.38) editado por M. Zanna. New York: Academic Press.

Watts, D. y P. S. Dodds. 2007. "Influentials, networks, and public opinion formation", Journal of Consumer Research 34: 441-458. https://doi.org/10.1086/518527

Watts, D. y P. S. Dodds. 2009. "Threshold models of social influence". Pp. 475497 en The Oxford Handbook of Analytical Sociology, editado por P. Hedström y P. Bearman. Oxford: Oxford University Press.

Weisbuch, G., G. Deffuant, F. Amblard y J. P. Nadal. 2002. "Meet, Discuss and Segregate!", Complexity 7(3):5563. https://doi.org/10.1002/cplx.10031

Weisbuch, G., G. Deffuant, F. Amblard y J. P. Nadal. 2003. "Interacting agents and continuous opinions dynamics". Pp. 225-242 en Heterogenous agents, interactions and economic performance, editado por R. Cowan y N. Jonard. Berlin, Heidelberg: Springer.

JORDI TENA-SÁNCHEZ es doctor en Sociología por la Universitat Autònoma de Barcelona (2010). Actualmente es Profesor Agregado en el Departamento de Sociología de dicha universidad y miembro del Grupo de Sociología Analítica y Diseño Institucional (GSADI). Su investigación se centra en aplicar los principios de la sociología analítica al estudio de diversos aspectos de las sociedades contemporáneas.

FRANCISCO J. LEÓN-MEDINA es doctor en Sociología por la Universitat Autònoma de Barcelona. Ha ejercido como docente en la Universitat Autònoma de Barcelona y la Universitat de Girona. Su investigación actual se centra en la filosofía de las ciencias sociales y en la ciencia social computacional, con un énfasis especial en el estudio de las dinámicas de la opinión pública mediante el uso de simulaciones multi-agente y Big Data. 